\title{
The Broad-specificity, Membrane-bound Lactate Dehydrogenase of Neisseria gonorrhoeae: Ties to Aromatic Metabolism
}

\author{
By RAJ K. BHATNAGAR, ${ }^{1}$ ANNE T. HENDRY, ${ }^{2} \mathrm{~K}$. T. SHANMUGAM ${ }^{1}$ \\ AND ROY A. JENSEN ${ }^{1 *}$ \\ ${ }^{1}$ Department of Microbiology and Cell Science, Institute of Food and Agricultural Sciences, \\ University of Florida, Gainesville, FL 32611, USA \\ ${ }^{2}$ Department of Laboratory Medicine, Hamilton General Hospital, 237 Barton Street East, \\ Hamilton, Ontario, Canada L8L $2 X 2$
}

(Received 2 August 1988; revised 24 October 1988; accepted 8 November 1988)

Neisseria gonorrhoeae can convert phenyllactate (PL) to phenylalanine and 4-hydroxyphenyllactate (HPL) to tyrosine. This was demonstrated by nutritional and physiological approaches. The enzymic basis for this unusual ability was shown to be the broad specificity of a particulate, unidirectional, pyridine-nucleotide-independent lactate dehydrogenase. This enzyme, denoted [iLDH], has been implicated in a pathogenic mechanism whereby host-derived lactate is linked to increased gonococcal oxygen consumption and electron transport. A similar role for HPL, a metabolite available in human host tissues, may provide a selective basis to explain evolution of broadened [iLDH] specificity in Neisseria. The interplay between aromatic metabolism and [iLDH] suggests new approaches for manipulating the host-pathogen relationship.

\section{INTRODUCTION}

Since the environment of the human mucosal surface is the only natural reservoir for the persistence of Neisseria gonorrhoeae, an intimate co-evolution of pathogenic capabilities in response to host-metabolism characteristics seems inevitable. The unexpected ability of $N$. gonorrhoeae to utilize phenyllactate (PL) and 4-hydroxyphenyllactate (HPL) as precursors of L-phenylalanine and L-tyrosine, respectively, raised the questions of whether this reflects a selective response to availability of these compounds from host metabolism and what the enzymic basis of these utilizations might be. Fortuitous insight about the metabolic fate of PL and HPL was gained via a curious phenomenon of gonococcal microbiology: the high frequency of naturally occurring metabolite-requiring auxotrophs (Catlin, 1973) and metabolite-sensitive variants (Hendry \& Dillan, 1984) in clinical isolates. Although the selective basis for such diversity of nutritional phenotype is not at all appreciated at present, an aromatic-amino-acidrequiring auxotroph and a phenylalanine-inhibited isolate have been key strains for demonstration of in vivo conversion of PL and HPL to L-phenylalanine and L-tyrosine, respectively. The broad specificity of a membrane-bound lactate dehydrogenase, [iLDH], was shown to be the enzymic catalyst for the foregoing conversions.

\section{METHODS}

Bacterial strains. Three isolates of Neisseria gonorrhoeae were used: (i) a typical isolate type that is resistant to growth inhibition by L-phenylalanine (ATCC 27630), (ii) a phenylalanine-sensitive, urogenital isolate

\footnotetext{
Abbreviations: PL, phenyllactate; HPL, 4-hydroxyphenyllactate; [iLDH], membrane-bound lactate dehydrogenase independent of pyridine nucleotide; [nLDH], soluble lactate dehydrogenase dependent upon pyridine nucleotide; MTT, 3-(4,5-dimethylthiazol-2-yl)-2,5-diphenyltetrazolium bromide.
} 
(ATCC 27628), and (iii) a clinical isolate (VHC 3102) obtained from Victoria Hospital Corporation (London, Ontario) having absolute requirements for L-phenylalanine, L-tyrosine and L-tryptophan.

Growth of bacteria. Strains were grown in a chemically defined medium (Hendry, 1983) to which supplements were added as indicated in the text. Cell mass was monitored as culture turbidity, using a Klett-Summerson colorimeter with a no. 66 filter. Exponentially growing cultures were used to inoculate $20 \mathrm{ml}$ fresh medium to give an initial density of 20-30 Klett units. These cultures were grown with vigorous shaking at $37^{\circ} \mathrm{C}$ in nephelometer flasks. Cell populations to be used as source of enzyme extract were grown in $2800 \mathrm{ml}$ Fernbach flasks containing 1 litre of medium. Cultures in the late exponential phase of growth were harvested by centrifugation, washed twice with $20 \mathrm{mM}$-potassium phosphate buffer, $\mathrm{pH} 7 \cdot 0$, containing $1 \mathrm{mM}$-dithiothreitol (DTT), and stored at $-80^{\circ} \mathrm{C}$ until used.

Agar disc diffusion methodology. The growth mass from 18-h-old cultures on Columbia base blood agar was suspended in inoculating fluid containing $0.05 \%$ fatty-acid-free albumin (Hendry, 1983). Sterile swabs were used to establish confluent lawns on the agar surface. Filter paper discs $(6 \mathrm{~mm}$ diameter) impregnated with nutrients as indicated in the legend to Fig. 1 were placed on the agar surface. The plates were incubated at $37^{\circ} \mathrm{C}$ under $6 \%(\mathrm{v} / \mathrm{v})$ $\mathrm{CO}_{2}$ and growth was observed after $24 \mathrm{~h}$ and $48 \mathrm{~h}$.

Preparation of cell-free extracts. Cell pellets were suspended in 20 mM-potassium phosphate buffer, $\mathrm{pH} 7 \cdot 0$, containing $1 \mathrm{mM}$-DTT and disrupted by two $30 \mathrm{~s}$ bursts of sonication (Labline Ultratip, $100 \mathrm{~W}$ output) with intermittent cooling. This crude preparation is designated as whole extract. The volume of the suspension was noted, and an aliquot was centrifuged at $20000 \mathrm{~g}$ for $30 \mathrm{~min}$. The supernatant, designated fraction $\mathrm{I}$, was saved. The remaining whole extract was divided into two equal portions. One portion was centrifuged at $150000 \mathrm{~g}$ for $1 \mathrm{~h}$. The supernatant (fraction III) was removed by means of a Pasteur pipette and saved. The pellet was resuspended uniformly in phosphate buffer by use of a teflon tissue homogenizer, and the membranes were solubilized by addition of $2 \%(\mathrm{v} / \mathrm{v})$ Triton $\mathrm{X}-100$ and $10 \mathrm{mM}-\mathrm{MgCl}_{2}$. The mixture was incubated at $25^{\circ} \mathrm{C}$ for $20 \mathrm{~min}$. The resulting suspension was centrifuged at $150000 \mathrm{~g}$ for $1 \mathrm{~h}$, and the supernatant, designated fraction IV, was saved. Membranes in the second aliquot of whole extract were also solubilized by incubation at $25^{\circ} \mathrm{C}$ for 20 min with $2 \%$ Triton X-100 and $10 \mathrm{mM}-\mathrm{MgCl}_{2}$. This was centrifuged at $20000 \mathrm{~g}$ for $30 \mathrm{~min}$. The supernatant, designated fraction II, was saved. All fractions I-IV were assayed for enzyme activities.

Ion-exchange chromatography. A portion of fraction IV (up to $30 \mathrm{mg}$ protein) was applied to a DEAE-cellulose (DE-52) column $(1.5 \times 10 \mathrm{~cm})$ equilibrated with $20 \mathrm{~mm}$-potassium phosphate buffer, $\mathrm{pH} 7.0$, containing $1 \mathrm{mM}$ DTT, $0.1 \%$ phenylmethylsulphonyl fluoride (PMSF), and 0.01 M-DL-lactic acid. Bound proteins were eluted at a flow rate of $30 \mathrm{ml} \mathrm{h}^{-1}$ with $150 \mathrm{ml}$ of a linear gradient $(0-0.50 \mathrm{M})$ of $\mathrm{KCl}$ prepared in equilibration buffer and fractions of $1.0 \mathrm{ml}$ were collected. Fractions showing lactate dehydrogenase activity were pooled and concentrated by use of a YM-10 ultrafiltration membrane, washed with phosphate buffer to remove the detergent, and used for enzyme assays.

Estimation of protein. Protein concentrations were estimated by the method of Bradford (1976).

Enzyme assays. Activity of [iLDH] was measured by phenazine-methosulphate-coupled reduction of MTT [3-(4,5-dimethylthiazol-2-yl)-2,5-diphenyltetrazolium bromide] by minor modifications to the procedure of Weiner \& Heppel (1972). To $1 \mathrm{ml}$ of standard assay mixture containing $0.1 \mathrm{M}$ phosphate buffer ( $\mathrm{pH} 7.5$ ) were added $60 \mu \mathrm{g}$ MTT ml-1, $120 \mu \mathrm{g}$ phenazine methosulphate $\mathrm{ml}^{-1}$, enzyme and substrate. In standard assays PL, HPL and lactate were used at a $5 \mathrm{~mm}$ concentration, a concentration which was saturating with any of the three substrates. Increase in absorbance at $570 \mathrm{~nm}$ at $25^{\circ} \mathrm{C}$ was monitored for $3 \mathrm{~min}$. Activity is expressed as nmol MTT reduced $\mathrm{min}^{-1}$ (mg protein)-1 using an absorption coefficient for MTT of $17 \mathrm{~mm}^{-1} \mathrm{~cm}^{-1}$ (Kistler \& Lin, 1971).

Pyridine-nucleotide-linked lactate dehydrogenase was assayed by the procedure of Holten \& Jyssum (1974). The reaction mixture in $1 \mathrm{ml}$ contained: $1 \mu \mathrm{mol} \mathrm{KCN}, 0.5 \mu \mathrm{mol}$ nicotinamide, $5 \mu \mathrm{mol}$ substrate, $0.25 \mu \mathrm{mol} \mathrm{NAD}^{+}$, $150 \mu \mathrm{mol}$ glycine $/ \mathrm{NaOH}$ buffer $(\mathrm{pH} 10.0)$ and an appropriate amount of protein. In the reverse reaction, $10 \mu \mathrm{mol}$ sodium pyruvate was used as substrate and $0-25 \mu \mathrm{mol}$ NADH as coenzyme in $110 \mu \mathrm{mol}$ potassium phosphate buffer ( $\mathrm{pH} 7$-4). Activity is expressed as nmol coenzyme reduced or oxidized $\mathrm{min}^{-1}$ (mg protein) ${ }^{-1}$.

Reduction of pyruvate, phenylpyruvate and 4-hydroxyphenylpyruvate by [iLDH] was assayed by following oxidation of reduced benzyl viologen at $550 \mathrm{~nm}$ (Spencer \& Guest, 1973) or of methyl viologen at $600 \mathrm{~nm}$ (Mizushima \& Kitahara, 1962). The reaction mixture in $2.0 \mathrm{ml}$ contained $20 \mu \mathrm{mol}$ substrate, $35 \mu \mathrm{mol}$ dye and $200 \mu \mathrm{mol}$ potassium phosphate buffer, $\mathrm{pH}$ 7.0. Reaction mixture tubes were flushed with nitrogen and a $1 \mathrm{~mm}$ solution of sodium dithionite was injected into the reaction tube to give an absorbance of $0 \cdot 8-1 \cdot 0$. Absorbance was followed for 1-2 min to measure any non-specific dye oxidation, and the reaction was then initiated by injection of enzyme protein (up to $4 \mathrm{mg}$ protein). Assay conditions of proportionality with respect to protein concentration and elapsed reaction time at saturating substrate concentration were used.

Isolation and identification of reaction products. Reaction mixtures in $20 \mathrm{ml}$ phosphate buffer containing $10 \mathrm{~mm}$ substrate and up to $25 \mathrm{mg}$ protein were incubated at $25^{\circ} \mathrm{C}$ for $20 \mathrm{~min}$. A $2.0 \mathrm{ml}$ sample of reaction mixture was then removed and treated with $0.5 \mathrm{ml} 20 \%(\mathrm{w} / \mathrm{v})$ metaphosphoric acid, and the clear supernatant was assayed for pyruvate, phenylpyruvate and 4-hydroxyphenylpyruvate by the method of Lin et al. (1958). The remaining 
reaction mixture was adjusted to $\mathrm{pH} 2.0$ with $\mathrm{HCl}$, and precipitated protein was removed by centrifugation. The clear supernatant was saturated with $\mathrm{NaCl}$ and extracted with diethyl ether $(3 \times 100 \mathrm{ml})$. The ether extract was dried with anhydrous $\mathrm{MgSO}_{4}$ and evaporated to dryness, then treated with 2,4-dinitrophenylhydrazine ( $0 \cdot 1 \%$ in $2 \mathrm{M}-\mathrm{HCl}$ ). The hydrazone derivatives were extracted into ethyl acetate and partially purified by extraction into $10 \%(\mathrm{w} / \mathrm{v}) \mathrm{Na}_{2} \mathrm{CO}_{3}$, acidification, and re-extraction into ethyl acetate. The hydrazone derivatives of the product and authentic standards were compared by thin-layer chromatography (Blakeley, 1977) on silica-coated plastic sheets (Eastman Kodak, 6060). The following solvent systems were used: (i) chloroform/methanol/water/formic acid $\left(100: 10: 9 \cdot 6: 0 \cdot 4\right.$, by vol.), and (ii) ethyl acetate (saturated with $\left.0 \cdot 1 \mathrm{M}-\mathrm{NaHCO}_{3}\right) / \mathrm{methanol}(5: 1, \mathrm{v} / \mathrm{v})$.

Polyacrylamide gel electrophoresis. This was done by the method of Laemmli (1970). After electrophoresis, the gel was cut longitudinally and stained for enzyme activity. The enzyme activity stain contained the following

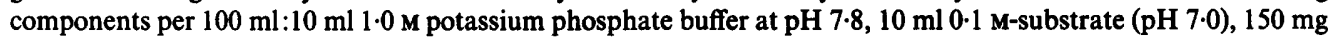
nitroblue tetrazolium, $40 \mathrm{mg}$ phenazine methosulphate, and $80 \mathrm{ml}$ water. Gels were incubated in the dark for $15 \mathrm{~min}$ at $25^{\circ} \mathrm{C}$.

\section{RESULTS}

Fig. 1 illustrates the unusual ability of the Aro- isolate VHC 3102 to satisfy its Lphenylalanine and L-tyrosine requirements with PL and HPL, respectively. These conversions appear to be generally unusual in bacteria. Thus, appropriate auxotrophs of Escherichia coli, Bacillus subtilis and Pseudomonas aeruginosa are unable to convert PL and HPL to phenylalanine and tyrosine (R. K. Bhatnagar, unpublished data). Fig. $1(a)$ illustrates the multiple requirement of VHC 3102 for L-tryptophan, L-tyrosine and L-phenylalanine, since growth occurs only at the diffusion interface of all three compounds. Fig. 1(b) shows a luxurious growth response at the diffusion interface of PL and HPL in the presence of L-tryptophan (in the agar medium). When the agar medium contained both L-tryptophan and L-tyrosine (Fig. 1c), discs containing PL supported a halo of growth that was at least as dense as those supported by discs containing phenylpyruvate or L-phenylalanine. Since isolate VHC 3102 has an absolute nutritional requirement for L-phenylalanine and L-tyrosine, it is clear that $\mathrm{PL}$ is an adequate precursor of $\mathrm{L}$ phenylalanine and that HPL is an adequate precursor of L-tyrosine in vivo.

A variety of other approaches yielded data supporting the foregoing conclusion. Fig. 2 illustrates a few of many experiments carried out in liquid medium. The herbicide glyphosate [ $N$-(phosphonomethyl)glycine] is a potent antimicrobial compound whose primary target of action is the sixth common-pathway enzyme of aromatic amino acid biosynthesis (Steinrücken \& Amrhein, 1980). Although aromatic amino acid supplementation did not totally prevent growth inhibition by glyphosate, probably a result of starvation for minor aromatic-pathway endproducts such as $p$-aminobenzoate or the result of an energy-drain effect described in bacteria by Fischer $e t$ al. (1986), PL and HPL were at least as effective as L-phenylalanine and Ltyrosine (Fig. 2a). Another approach, illustrated by data shown in Fig. 2(b), exploited the sensitivity of isolate ATCC 27628 to growth inhibition in the presence of L-phenylalanine (Hendry \& Dillon, 1984; Berry et al., 1987), i.e. demonstrating sensitivity to growth inhibition by PL as well. Isolate ATCC 27628 is also sensitive to growth inhibition by phenylpyruvate (Hendry \& Dillon, 1984). Since the kinetics of inhibition by PL resemble the kinetics of inhibition by phenylpyruvate more than those of inhibition by L-phenylalanine, conversion of PL to phenylpyruvate is implicated as a first step of PL transformation to L-phenylalanine. The results in Fig. 2(b) show that L-tyrosine (or HPL, unpublished data) prevent (addition at zero time) or reverse (addition at $4 \mathrm{~h}$ ) PL-mediated inhibition of growth, exactly as found when inhibition was caused by phenylpyruvate or L-phenylalanine.

Still other experiments indicate excellent ability of HPL to replace L-tyrosine and of PL to replace L-phenylalanine. For example, growth inhibition of isolate ATCC 27630 by tyrosine analogues such as $m$-fluorotyrosine is reversed as well or better by HPL as by L-tyrosine. Similarly, growth inhibition by phenylalanine analogues, such as $m$-fluorophenylalanine, is reversed as well or better by PL than by L-phenylalanine.

The few cases reported of microbial ability to transform PL to phenylpyruvate include an $\mathrm{NAD}^{+}$-dependent hydroxyisocaproate dehydrogenase in Lactobacillus casei (Schmidt et al., 1987) and an NAD ${ }^{+}$-dependent D-lactate dehydrogenase in Lactobacillus confusus (Schütte et al., 

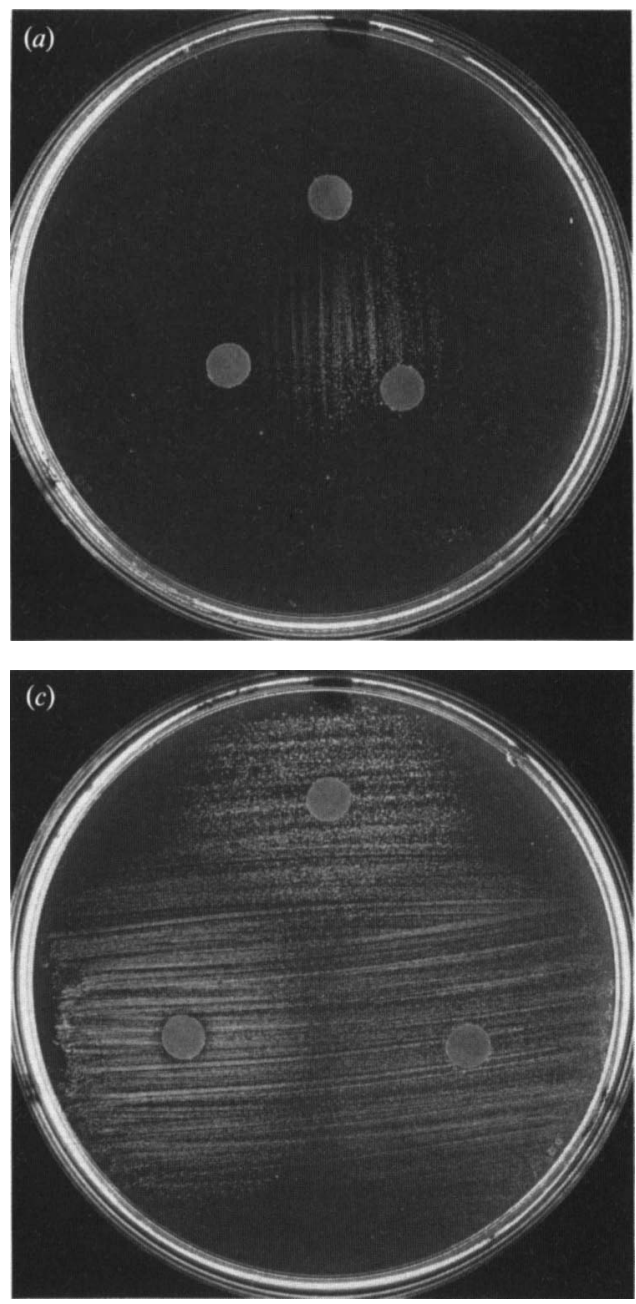

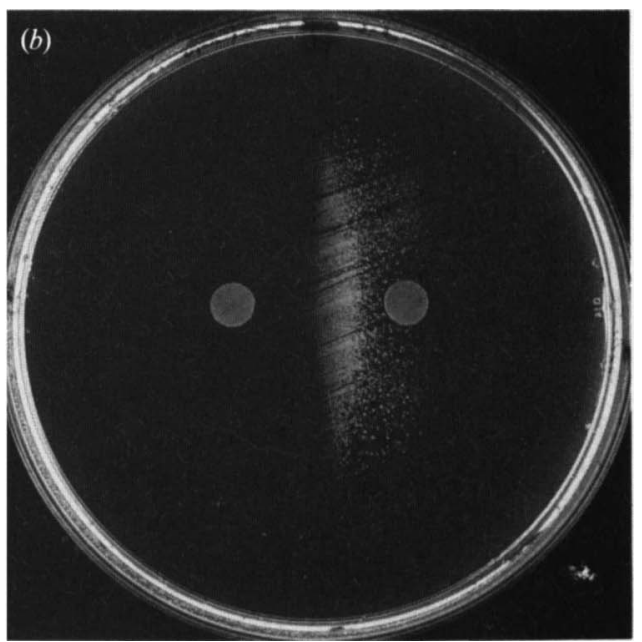

Fig. 1. Ability of isolate VHC 3102 to substitute HPL and PL for tyrosine and phenylalanine, respectively. Isolate VHC 3102 requires uracil and L-arginine for growth, and all media were appropriately supplemented with these compounds. Photographs shown are the results of an agar-disc diffusion technique in which agar plates were spread with confluent lawns of isolate VHC 3102. Sterile $6 \mathrm{~mm}$ paper discs contained nutrients as indicated (amount per disc): L-tryptophan, $0.08 \mu \mathrm{mol}$; L-tyrosine, $0.4 \mu \mathrm{mol}$; L-phenylalanine, $0.4 \mu \mathrm{mol}$; L-phenyllactate (PL), $0.4 \mu \mathrm{mol}$; DL-4-hydroxyphenyllactate (HPL), $0.4 \mu \mathrm{mol}$; phenylpyruvate, $0.4 \mu \mathrm{mol}$; and 4 -hydroxyphenylpyruvate, $0.2 \mu \mathrm{mol}$. (a) The top, left and right discs contained L-tryptophan, L-phenylalanine and L-tyrosine, respectively. (b) The agar medium was made up with $0.5 \mathrm{mM}$ L-tryptophan, and the left and right discs contained PL and HPL, respectively. (c) The agar medium was made up with $0.05 \mathrm{mM}$-L-tryptophan and 0.25 mM-L-tyrosine. The top, left and right discs contained $L$-phenylalanine, PL and phenylpyruvate, respectively. Photograph $(c)$ was taken after $48 \mathrm{~h}$ incubation $\left(37^{\circ} \mathrm{C}\right.$ in the presence of $\left.6 \% \mathrm{CO}_{2}\right)$; the other photographs were taken after $24 \mathrm{~h}$.

1984). However, in a series of preliminary experiments, we found no indication of enzymic ability by $N$. gonorrhoeae extracts to oxidize PL or HPL in the presence of NAD ${ }^{+}$or NADP ${ }^{+}$. An NADH-specific lactate dehydrogenase, denoted [nLDH] (Garvie, 1980), was observed as a soluble enzyme (Table 1). No enzyme activity was found when PL or HPL was substituted for lactate as substrate. Since the $[\mathrm{nLDH}]$ prefers the oxoacid substrate by almost two orders of magnitude (Table 1), we tested phenylpyruvate and 4-hydroxyphenylpyruvate as substrates in combination with NADH in order to maximize detection of the substrate ambiguity sought. No activity was found.

Since particulate, membrane-associated lactate dehydrogenase species that are pyridine nucleotide independent ([iLDH]) are well known (Garvie, 1980), we pursued the possibility, using the phenazine-methosulphate-coupled reduction of MTT method (Weiner \& Heppel, 1972), that a broad-specificity [iLDH] might be responsible for oxidation of PL and HPL. The results in Table 1 show that a very active [iLDH] species was present in the particulate fraction 


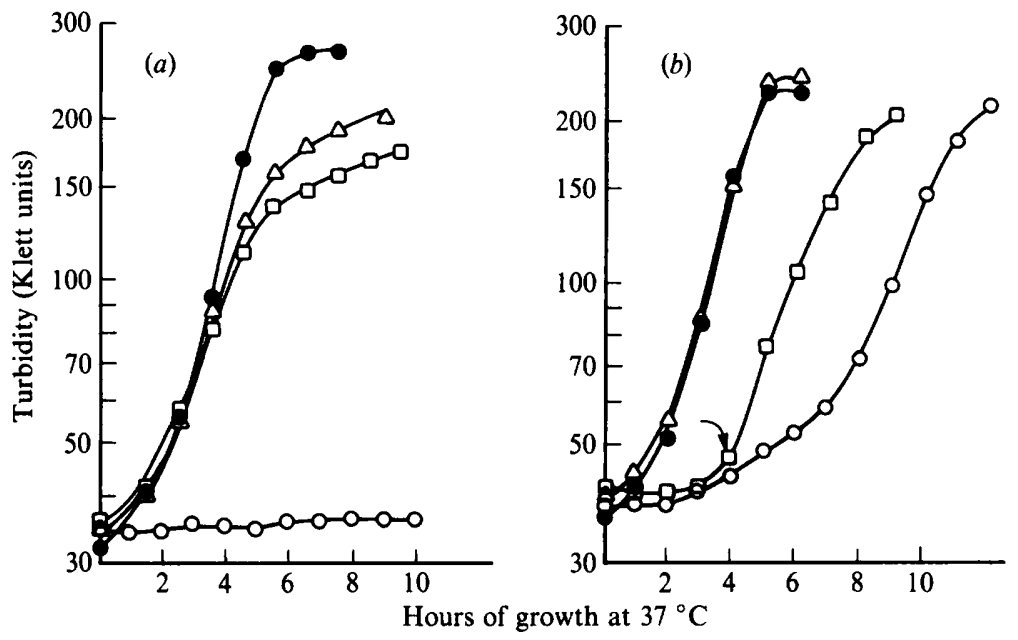

Fig. 2. Ability of PL and HPL to replace L-phenylalanine and L-tyrosine, respectively, as nutrients in a chemically defined liquid medium (Hendry, 1983). (a) Isolate ATCC 27630 is a proline-requiring auxotroph, and all growth flasks contained $2 \mathrm{mM}$-proline. The uninhibited rate of growth $(O)$ can be compared to inhibition in the presence of $100 \mu \mathrm{g}$ glyphosate $\mathrm{ml}^{-1}(\mathrm{O})$. Other growth flasks contained $100 \mu \mathrm{g} \mathrm{ml}^{-1}$ each of glyphosate, L-phenylalanine, L-tyrosine and L-tryptophan ( $\square$ ), or $100 \mu \mathrm{g} \mathrm{ml}^{-1}$ each of glyphosate, PL, HPL and L-tryptophan $(\Delta)$. (b) Isolate ATCC 27628 has no growth requirements (O) but its growth is inhibited by L-phenylalanine or phenylpyruvate (Hendry \& Dillon, 1984). O, Growth inhibition by $30 \mu \mathrm{g} \mathrm{PL} \mathrm{ml} l^{-1} . \triangle$, Growth in medium containing $30 \mu \mathrm{g} \mathrm{PL} \mathrm{ml}^{-1}+50 \mu \mathrm{g} \mathrm{L}$-tyrosine ml $^{-1}$. $\square$, Growth in medium containing $30 \mu \mathrm{g} \mathrm{PL} \mathrm{ml} \mathrm{m}^{-1}$ added at time zero, with $50 \mu \mathrm{g} \mathrm{L}$-tyrosine $\mathrm{ml}^{-1}$ added at $4 \mathrm{~h}$ (arrow). The results shown are representative of three experiments that gave very similar results.

Table 1. Fractionation of soluble and particulate lactate dehydrogenases

Crude extract prepared as described in Methods was centrifuged at $20000 \mathrm{~g}$ for $20 \mathrm{~min}$ to yield a supernatant (fraction I). An aliquot of crude extract was solubilized with detergent as described in Methods to give fraction II. Fraction I was centrifuged at $150000 \mathrm{~g}$ for $60 \mathrm{~min}$ to give a supernatant designated fraction III. The $150000 \mathrm{~g}$ pellet was solubilized as described in Methods to yield fraction IV.

Fraction

I $20000 \mathrm{~g}$ supernatant

II Whole extract, Triton X-100

III $150000 \mathrm{~g}$ supernatant

IV $150000 \mathrm{~g}$ pellet, Triton X-100

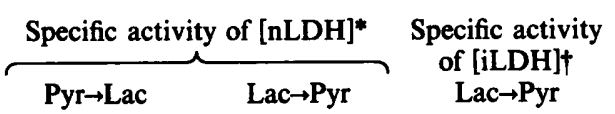

$24 \cdot 0$
0.4

0.5
$5 \cdot 2$

$242 \cdot 3$
Total units of $[\mathrm{iLDH}] \ddagger$

- Specific activity is expressed as nmol NADH (or NAD ${ }^{+}$) $\mathrm{min}^{-1}$ (mg protein) ${ }^{-1}$. Pyr, pyruvate; Lac, lactate. + Specific activity is expressed as nmol MTT reduced min $^{-1}$ (mg protein $)^{-1}$.

$\ddagger$ One unit is one nmol MTT reduced $\mathrm{min}^{-1}$.

obtained after centrifugation at $150000 \mathrm{~g}$. After solubilizing the membrane proteins with Triton $\mathrm{X}-100$, [iLDH] was purified about 46 -fold with about $90 \%$ recovery, judging from the [iLDH] levels measured after solubilization of the crude extract. When PL and HPL were tested as alternative substrates for lactate, specific activities of 55.5 for either substrate were obtained, using fraction-IV preparations. Roughly the same activity ratios were obtained with fraction-II preparations, although activities with PL or HPL as substrate were near the limits of detection. Thus, PL and HPL are about $22 \%$ as effective as lactate in function as substrate molecules. Apparent $K_{\mathrm{m}}$ values calculated for lactate, PL and HPL (DL-mixtures) were $0.08 \mathrm{mM}, 0.33 \mathrm{mM}$ 


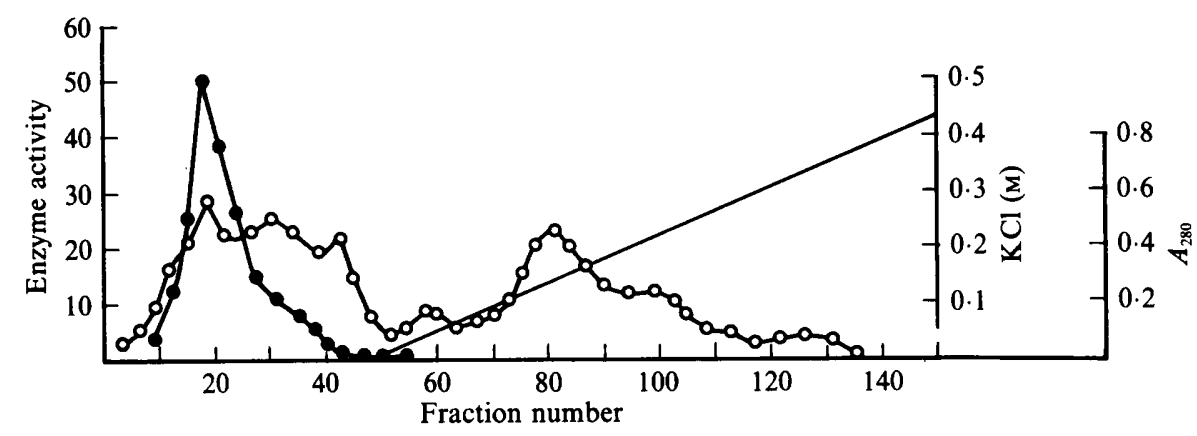

Fig. 3. Elution profile from DEAE-cellulose of [iLDH] from $N$. gonorrhoeae VHC 3102. Essentially identical results were obtained with ATCC 27630 and ATCC 27628. Fractionation of solubilized protein, and assay of [iLDH], are described in Methods. O, Protein profile $\left(A_{280}\right)$;, enzyme activity (nmol MTT reduced $\min ^{-1}$ per $100 \mu \mathrm{l}$ ); the diagonal line represents the linear $\mathrm{KCl}$ gradient.

and $0.33 \mathrm{~mm}$, respectively. The solubilized [iLDH] was quite unstable at $0-4{ }^{\circ} \mathrm{C}$. Inclusion of $10 \mathrm{mM}$-DL-lactic acid and $0.1 \%$ PMSF in the extraction buffer stabilized the activity only partially. Direct confirmation of the formation of 4-hydroxyphenylpyruvate and phenylpyruvate from HPL and PL, respectively, was obtained by preparation of 2,4-dinitrophenylhydrazone derivatives followed by resolution via thin layer chromatography (Blakeley, 1977), or by use of an enol-borate analytical procedure (Lin et al., 1958).

A procedure for following the oxidation of a reduced artificial dye system was used to determine whether pyruvate, phenylpyruvate or 4-hydroxyphenylpyruvate could be reduced by the enzyme. Activity with pyruvate was more than 150-fold less than that with lactate, and no activity was detected with phenylpyruvate or 4-hydroxyphenylpyruvate. Thus, the [iLDH] enzyme is essentially unidirectional.

Polyacrylamide gel electrophoresis of the solubilized membrane preparations yielded multiple protein bands, a single major band being reactive with all three substrates (lactate, PL and HPL). D-Lactate was $30 \%$ as effective as L-lactate, and again activity with each isomer migrated to the same single band position. When solubilized preparations of [iLDH] were further purified by ion-exchange chromatography, a single peak of activity was eluted (Fig. 3). This preparation of [iLDH] possessed all of the aforementioned properties, including the same activity ratios with PL, HPL and lactate. Thus, a single species of [iLDH], lacking absolute stereospecificity but preferring the L-isomer, appears responsible for catalysis with lactate, PL and HPL. The [iLDH] enzyme reported here corresponds to the pyridine-nucleotide-independent enzyme described previously (Johnston \& Gotschlich, 1974). The joint presence of [nLDH] and [iLDH] in $N$. gonorrhoeae has been known for some time (see Morse, 1979).

\section{DISCUSSION}

Membrane-associated lactate dehydrogenase has been linked to the operation of the electrontransport chain in Gram-negative bacteria such as Escherichia coli (Kaback \& Barnes, 1971) and Pseudomonas aeruginosa (Kemp, 1972). The [iLDH] of $N$. gonorrhoeae has also been implicated as a key entity of the electron-transport chain (Winter \& Morse, 1975).

A possible linkage between [iLDH], aromatic metabolism and a biochemical aspect of pathogenesis is formulated on the basis of the following considerations. Lactic acid, a common metabolite of human biological fluids, is readily utilized as one of the few carbon and energy sources within the limited carbohydrate repertoire of $N$. gonorrhoeae (Morse, 1979). The oxidation of lactate is coupled to electron transport via membrane-bound [iLDH]. A case has been made (Britigan et al., 1988) that lactate derived from a host origin (e.g. neutrophil glucose catabolism) drives gonococcal oxygen consumption and growth. The resulting near-anaerobic conditions create redox conditions that are favourable for continued proliferation of the pathogen. Although $N$. gonorrhoeae has until recently been described as an aerobic organism 
containing high levels of cyctochrome $c$ oxidase, the foregoing statement is tenable in view of the results obtained by Knapp \& Clark (1984). They showed that $N$. gonorrhoeae grows well anaerobically with use of nitrite as a terminal electron acceptor. Nitrite reductase is produced constitutively by gonococci, and $N$. gonorrhoeae is apparently able to make facile physiological transitions between anaerobic and aerobic states of growth. Nitrite is present in biological fluids, and a strong case was made (Knapp \& Clark, 1984) for ability of $N$. gonorrhoeae to grow anaerobically in the cervix, rectum and oropharynx as sites of colonization - a conclusion consistent with the presence of co-existing anaerobes in these environments. The [iLDH] has an unusual breadth of substrate recognition that results in utilization of both PL and HPL. Although significant levels of PL may only be present in biological fluids under abnormal host conditions such as found in phenylketonuria, scurvy and tyrosinaemia, and in premature infants, HPL is present in normal host tissues. Thus, HPL is an intermediate of ubiquinone biosynthesis; the breakdown of catecholamines yields HPL in amniotic fluid (Lentner, 1981, p. 90); and HPL is present at $0 \cdot 3-2 \cdot 2 \mathrm{nmol}$ per mol creatinine in urine (Lentner, 1981, p. 207).

The unusually broad specificity of $N$. gonorrhoeae [iLDH] suggests a selective advantage conferred by ability to oxidize a variety of lactyl derivatives (or at least HPL) encountered in the host-pathogen environment. Thus, oxidation of HPL would potentially: (i) initiate membrane energization in electron transport, (ii) tend to create more anaerobic conditions that disfavour the effectiveness of host neutrophils, and (iii) provide a source of tyrosine, an expensive metabolite to synthesize and apparently not necessarily available in excess from the host since $N$. gonorrhoeae possesses a complete biosynthetic pathway (Berry et al., 1987). This broadspecificity [iLDH] appears to be a trait shared by other Neisseria species, since $N$. meningitidis, $N$. lactamica, $N$. mucosa and $N$. sicca were all able to replace L-tyrosine with HPL as an effective antagonist of the growth-inhibitory effects of $m$-fluorotyrosine (A. T. Hendry, unpublished data). If [iLDH] proves to play an essential role in the host-pathogen relationship, it must be a necessary-but-not-sufficient component since the non-pathogenic Neisseria species appear to possess a similar [iLDH] enzyme.

The substrate ambiguity of [iLDH] provides a novel basis for selection of [iLDH] mutants (e.g. retention of the ability of L-tyrosine, but not of HPL, to reverse growth inhibition by $m$ fluorotyrosine). A new basis for the design of inhibitor agents that block [iLDH] function is also established.

This research was supported in part by NIH grant AM38309 to R.A.J. This paper is Florida Agricultural Station Journal Series no. 9186.

\section{REFERENCES}

Berry, A., Jensen, R. A. \& Hendry, A. T. (1987). Enzymatic arrangement and allosteric regulation of the aromatic amino acid pathway in Neisseria gonorrhoeae. Archives of Microbiology 149, 87-94.

BLAKELEY, E. R. (1977). The catabolism of L-tyrosine by an Arthrobacter sp. Canadian Journal of Microbiology 23, 1128-1139.

BRADFORD, M. M. (1976). A rapid and sensitive method for the quantitation of microgram quantities of protein utilizing the principle of protein-dye binding. Analytical Biochemistry 72, 248-254.

Britigan, B. E., Klapper, D., Svendsen, T. \& CohEN, M. S. (1988). Phagocyte-derived lactate stimulates oxygen consumption by Neisseria gonorrhoeae, an unrecognized aspect of the oxygen metabolism of phagocytosis. Journal of Clinical Investigation 81, 318-324.

Catlin, B. W. (1973). Nutrition profiles of Neisseria gonorrhoeae, Neisseria meningitidis, and Neisseria lactamica in chemically defined media and the use of growth requirements for gonococcal typing. Journal of Infectious Diseases 128, 178-194.
Fischer, R., Berry, A., Gaines, C. \& Jensen, R. A. (1986). Comparative action of glyphosate as a trigger of energy drain in eubacteria. Journal of Bacteriology 168, 1147-1154.

GARVIE, E. (1980). Bacterial lactate dehydrogenases. Microbiological Reviews 44, 106-139.

HeNDRY, A. T. (1983). Growth responses of Neisseria gonorrhoeae auxotypes to required amino acids and bases in liquid medium. Canadian Journal of Microbiology 29, 1309-1313.

HeNDRY, A. T. \& Dillon, J. R. (1984). Growth inhibition of Neisseria gonorrhoeae isolates by Lphenylalanine and its analogues in defined media. Canadian Journal of Microbiology 30, 1319-1325.

Holten, E. \& JYssum, K. (1974). Activities of some enzymes concerning pyruvate metabolism in Neisseria. Acta pathologica et microbiologica scandinavica B82, 843-848.

Johnston, K. H. \& Gotschlich, E. C. (1974). Isolation and characterization of the outer membrane of Neisseria. Journal of Bacteriology 119, 250257. 
KABACK, H. R. \& BARNES, E. M. (1971). Mechanisms of active transport in isolated membrane vesicles. The mechanism of energy coupling between D-lactic dehydrogenase and $\beta$-galactoside transport in membrane preparations from Escherichia coli. Journal of Biological Chemistry 246, 5523-5531.

KEMP, M. B. (1972). D- and L-lactate dehydrogenase of Pseudomonas aeruginosa. Biochemical Journal 130, 307-309.

KistleR, W. S. \& LiN, E. C. C. (1971). Anaerobic L- $\alpha-$ glycerophosphate dehydrogenase of Escherichia coli: its genetic locus and its physiological role. Journal of Bacteriology 108, 1224-1234.

KNAPP, J. S. \& CLARK, V. L. (1984). Anaerobic growth of Neisseria gonorrhoeae coupled to nitrite reduction. Infection and Immunity 46, 176-181.

LAEMMLI, U. K. (1970). Cleavage of structural proteins during the assembly of the head of bacteriophage T4. Nature, London 227, 680-685.

LENTNER, C. (editor) (1981). Geigy Scientific Tables, Vol. 1. Basle: Ciba-Geigy.

Lin, E. C. C., Pitt, B. M., Morton, C. \& Knox, E. W. (1958). The assay of aromatic amino acid transamination and keto acid oxidation by the enol-borate tautomerase method. Journal of Biological Chemistry 233, 668-673.

Mizushima, S. \& Kitahara, K. (1962). Purification and properties of lactic dehydrogenase of Lactobacillus casei. Journal of General and Applied Microbiology 8, 130-141.
Morse, S. A. (1979). The biology of the gonococcus. Critical Reviews in Microbiology 7, 93-189.

SCHMIDT, E., VASIC-RACKI, D. \& WANDREY, C. (1987). Enzymatic production of L-phenylalanine from the racemic mixture of $\mathrm{D}, \mathrm{L}-$ phenyllactate. Applied and Microbiological Biotechnology 26, 42-48.

SCHüTTe, H., HuMmel, W. \& KulA, M.-R. (1984). L-2Hydroxyisocaproate dehydrogenase - a new enzyme from Lactobacillus confusus for the stereo-specific reduction of 2-ketocarboxylic acids. Applied and Microbiological Biotechnology 19, 167-176.

STEINRÜCKEN, H. C. \& AMRHEIN, N. (1980). The herbicide glyphosate is a potent inhibitor of 5-enolpyruvylshikimic acid 3-phosphate synthase. Biochemical and Biophysical Research Communications 94, 1207-1212.

SPENCER, M. E. \& Guest, J. R. (1973). Isolation and properties of fumarate reductase mutants of Escherichia coli. Journal of Bacteriology 114, 563-570.

Weiner, J. H. \& HePpel, L. A. (1972). Purification of the membrane-bound pyridine nucleotide independent L-glycerol-3-phosphate dehydrogenase from Escherichia coli. Biochemical and Biophysical Research Communications 47, 1360-1365.

Winter, B. D. \& Morse, S. A. (1975). Physiology and metabolism of pathogenic Neisseria : partial characterization of the respiratory chain of Neisseria gonorrhoeae. Journal of Bacteriology 123, 631-636. 